\title{
Computational investigation of potential dosing schedules for a switch of medication from warfarin to rivaroxaban - an oral, direct Factor Xa inhibitor
}

\author{
Rolf Burghaus ${ }^{1}$, Katrin Coboeken ${ }^{2}$, Thomas Gaub ${ }^{2}$, Christoph Niederalt ${ }^{2}$, Anke Sensse ${ }^{1}$, \\ Hans-Ulrich Siegmund ${ }^{2}$, Wolfgang Weiss ${ }^{2}$, Wolfgang Mueck ${ }^{1}$, Takahiko Tanigawa ${ }^{1}$ and Jörg Lippert ${ }^{1 *}$ \\ 'Bayer HealthCare, Wuppertal, Germany \\ 2 Bayer Technology Services GmbH, Leverkusen, Germany
}

Edited by:

Raimond L. Winslow, The Johns

Hopkins University, USA

Reviewed by:

Mary Margot Catherine Maleckar,

Simula Research Laboratory,

Norway

Manash Shankar Chatterjee, Merck

\& Co, USA

${ }^{*}$ Correspondence:

Jörg Lippert, Bayer HealthCare,

Clinical Pharmacometrics, Geb. 431,

42113 Wuppertal, Germany

e-mail: joerg.lippert@bayer.com
The long-lasting anticoagulant effect of vitamin $K$ antagonists can be problematic in cases of adverse drug reactions or when patients are switched to another anticoagulant therapy. The objective of this study was to examine in silico the anticoagulant effect of rivaroxaban, an oral, direct Factor Xa inhibitor, combined with the residual effect of discontinued warfarin. Our simulations were based on the recommended anticoagulant dosing regimen for stroke prevention in patients with atrial fibrillation. The effects of the combination of discontinued warfarin plus rivaroxaban were simulated using an extended version of a previously validated blood coagulation computer model. A strong synergistic effect of the two distinct mechanisms of action was observed in the first 2-3 days after warfarin discontinuation; thereafter, the effect was close to additive. Nomograms for the introduction of rivaroxaban therapy after warfarin discontinuation were derived for Caucasian and Japanese patients using safety and efficacy criteria described previously, together with the coagulation model. The findings of our study provide a mechanistic pharmacologic rationale for dosing schedules during the therapy switch from warfarin to rivaroxaban and support the switching strategies as outlined in the Summary of Product Characteristics and Prescribing Information for rivaroxaban.

Keywords: coagulation, combination therapy, mathematical modeling, pharmacodynamics, rivaroxaban, simulation, warfarin

\section{INTRODUCTION}

Many patients who require long-term vitamin $\mathrm{K}$ antagonist (VKA; e.g., warfarin) therapy experience difficulties in maintaining a therapeutic international normalized ratio (INR) (Ageno et al., 2012). Some patients may benefit from switching to one of the direct oral anticoagulants, such as rivaroxaban, which have been approved for the management of several thromboembolic disorders, including the prevention of stroke and systemic embolism in adult patients with non-valvular atrial fibrillation and at least one other risk factor for stroke. Rivaroxaban has been approved in this indication in Europe and the United States (at a dose of $20 \mathrm{mg}$ once daily) (Bayer Pharma, 2014; Janssen Pharmaceuticals Inc., 2014) and in Japan (at a dose of $15 \mathrm{mg}$ once daily) (Bayer Yakuhin Ltd., 2012; Hori et al., 2012).

VKAs are indirect anticoagulants that target multiple enzymes in the coagulation cascade. Specifically, they inhibit vitamin K-dependent clotting factors including Factors II, VII, IX, and $\mathrm{X}$, and they also inhibit the carboxylation of the anticoagulant proteins $\mathrm{C}$ and $\mathrm{S}$; therefore, VKAs have the potential to be procoagulants (Ansell et al., 2008). The use of VKAs is associated with the need for frequent monitoring and dose adjustment (Ansell et al., 2008) to ensure that the INR reaches and remains within the recommended therapeutic range (Ansell et al., 2008; Douketis et al., 2008; Kearon et al., 2012). The INR, based on the prothrombin time (PT) coagulation test, was developed to provide a standardized measure of the anticoagulant effect of VKAs and is the basis of guidelines for warfarin therapy (Ageno et al., 2012). Target INR values established for VKA therapies are specific to the distinct mechanisms of action of these agents and cannot be translated to anticoagulants with different mechanisms of action. For example, INR values measured in the therapeutic range of rivaroxaban are significantly lower than those required under warfarin therapy, indicating that INR measurements are not valid in this case (Kubitza et al., 2005a,b; Ansell et al., 2008; Douketis et al., 2008; Kearon et al., 2012).

Dose-response relationships of VKAs may be influenced by genetic factors, drug-drug interactions, and the consumption of alcohol and foods containing vitamin K (Ansell et al., 2008). For example, mutations in the vitamin $\mathrm{K}$ oxide reductase gene lead to enzymes with varying sensitivities to warfarin inhibition. Different ethnic populations vary in the frequency with which these mutations occur and, therefore, require different warfarin doses to maintain a therapeutic INR.

After discontinuation of warfarin, the concentrations of active vitamin K-dependent clotting factors and anticoagulant proteins $\mathrm{C}$ and $\mathrm{S}$ increase slowly toward normal levels. Because all of these factors are generated and cleared slowly, coagulation behavior is normalized only several days after warfarin discontinuation. 
Therefore, transitioning between warfarin and rivaroxaban therapies must be managed carefully and must account for the slow onset and long washout of warfarin effects to minimize the risk of bleeding and thrombotic events (Faaij et al., 2001, 2002; Reiffel, 2004; Patel et al., 2011).

Rivaroxaban is an oral, direct Factor Xa inhibitor with a wide therapeutic window (Perzborn et al., 2005; Roehrig et al., 2005) and predictable pharmacokinetic $(\mathrm{PK})$ and pharmacodynamic (PD) properties. Because of its mechanism of action, rivaroxaban influences routine coagulation assays such as the PT/INR and activated partial thromboplastin time (aPTT). Phase I studies have shown a close correlation between PT and rivaroxaban plasma levels (Kubitza et al., 2005a,b).

In a previous study, we used an ordinary differential equation (ODE)-based computer model for blood coagulation to assess the optimal balance between efficacy and safety scenarios for rivaroxaban (Burghaus et al., 2011). This model was based on prior knowledge about coagulation factor concentrations after anticoagulant administration and data from ex vivo clotting tests; the model structurally resembles other recently published models of the effect of rivaroxaban on coagulation (Orfeo et al., 2010, 2011).

When modeling coagulation behavior during the transition phase from warfarin to rivaroxaban, the long PK and PD decay of warfarin, in conjunction with the effect of both warfarin and rivaroxaban on coagulation characteristics such as PT, need to be considered.

Currently, there is a lack of clinical data regarding PD changes during the transition from VKA therapy to direct oral anticoagulants, including rivaroxaban. Therefore, data derived from modeling approaches will provide useful information for clinicians planning to investigate the switch of medication experimentally.

The computational model used in this study was composed of the coagulation model as published by Burghaus et al. (2011), and the warfarin decay model describing the time course of coagulation factor concentrations as a result of warfarin treatment or its discontinuation. This model was used to investigate changes in the combined coagulation effect during the switch from warfarin to rivaroxaban. Our objectives were to improve mechanistic understanding of the interaction between warfarin and rivaroxaban during the transition and to provide a mechanistic pharmacologic rationale for dosing schedules during the therapy switch, thereby supporting the switching strategies as outlined in the Summary of Product Characteristics and Prescribing Information for rivaroxaban (Bayer Pharma, 2014; Janssen Pharmaceuticals Inc., 2014).

\section{MATERIALS AND METHODS MODEL SETUP}

We simulated the effect of warfarin monotherapy and the combined effects of warfarin and rivaroxaban using an ODE-based blood coagulation model that represents coagulation in ex vivo clotting tests with human plasma such as PT and aPTT; the model has been described previously (Burghaus et al., 2011) and is based largely on several published models (Kogan et al., 2001; Hockin et al., 2002; Anand et al., 2003; Bungay et al., 2003; Orfeo and Mann, 2005). The model, as described previously by Burghaus et al. (2011), takes into account both the intrinsic and extrinsic pathways of the coagulation cascade, as well as the common pathways leading to fibrin generation via thrombin (Figure 1). As such, it possesses some unique features that were not included in earlier models, such as a portfolio of drug action mechanisms. Study drugs were modeled by closely representing their anticoagulant properties (Burghaus et al., 2011). Supplementary Material 1-Model Pack, provides the complete model as implemented in $\mathrm{MoBi}^{\circledR}$ and all (Matlab ${ }^{\circledR}$ ) codes used for simulations and generation of figures. This provides full insight into the processes of computational warfarin titration and the parameters used for the simulations.

By use of parameter studies, the model can be used to investigate scenarios of blood coagulation initiated by very weak triggers that cannot be investigated in a controlled manner in experimental assays. For the simulations in this study we used the previously described model and added a turnover model for the synthesis and decay of vitamin K-dependent Factors II, VII, IX, and X, and proteins $\mathrm{C}$ and $\mathrm{S}$. Steady-state concentrations of these factors were set as previously reported in Burghaus et al. (2011). In the current study, model parameters that correspond to blood flow were set to zero.

The mechanism of action of rivaroxaban was already implemented in the previously described model (Burghaus et al., 2011). However, warfarin action had to be newly implemented: warfarin PK was not explicitly modeled. Both the steady-state effect of warfarin and its decay after discontinuation of therapy are represented by effective inhibition of synthesis rates of vitamin K-dependent coagulation factors in a standard turnover model. Parameters of the turnover model were chosen such that steady-state conditions (starting concentrations) for vitamin K-dependent factors of the turnover model corresponded to previously published data (Wittkowsky, 2003). No PK interactions between warfarin and rivaroxaban have been identified and, therefore, such potential interactions were not accounted for in the model (Food and Drug Administration, 2011; Johnson and Johnson Pharmaceutical Research and Development, 2011).

\section{Turnover model for vitamin K-dependent coagulation factors}

The turnover model for vitamin K-dependent coagulation factors (Table 1) describes the formation and clearance of the relevant proteins. Coagulation factor clearance is implemented as an exponential decay to reflect physiological conditions. The half-lives used in the model were as follows (Table 1C): Factor II, $57 \mathrm{~h}$; Factor VII, 5 h; Factor IX, 25.5 h; Factor X, 37.5 h; protein C, 9 h; protein S, 60 h (Wittkowsky, 2003).

Therapeutic warfarin is considered to be applied in a racemic 1:1 mixture of R-warfarin and S-warfarin. The effect of both warfarin components was implemented as a pre-factor $\mathrm{W}$ reducing the formation rates of coagulation factors according to the following equation, with $\mathrm{R}$-warfarin and S-warfarin representing the respective effective concentrations (in arbitrary units; see below and Supplementary Material 1):

$$
\mathrm{W}=\left([\mathrm{R} \text {-warfarin }+3.25 \times \mathrm{S} \text {-warfarin }]^{2}+1\right)^{-1}
$$

This equation takes into account that $\mathrm{S}$-warfarin is, on average, 3.25-fold more potent than R-warfarin, with the square having 


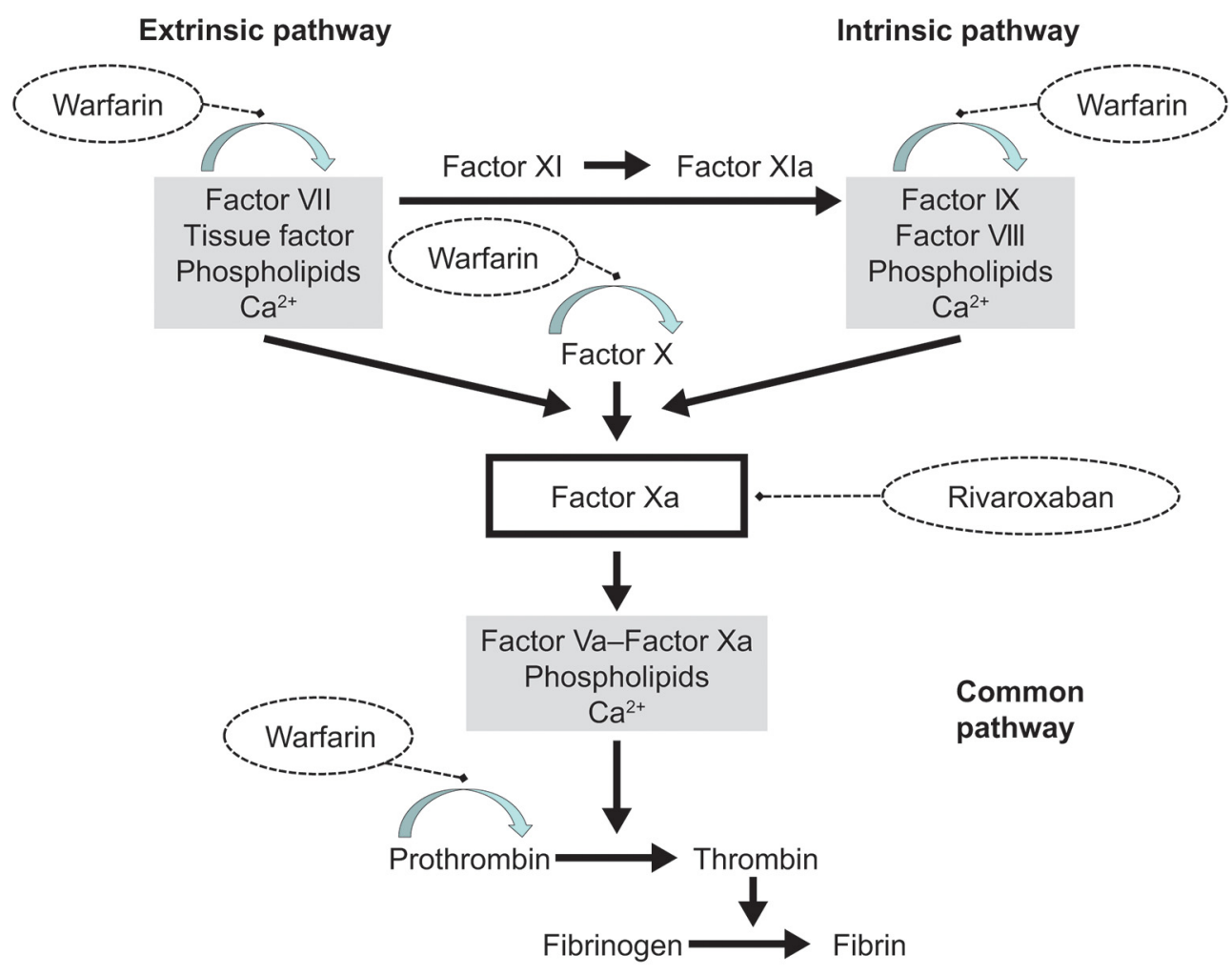

FIGURE 1 | Overview of the processes accounted for by the coagulation model. The blood coagulation model represents the biochemical reactions that result in factor activation. The extrinsic and intrinsic pathways of the model lead to thrombin and fibrin formation downstream of Factor Xa, known as the common pathway. Drug action is represented by the competitive inhibition of Factor $\mathrm{Xa}$ by rivaroxaban and by down-regulation of the vitamin K-dependent synthesis (arched arrow) of the factors VII, IX, and X, and prothrombin (Factor II) by warfarin. These structural elements of the coagulation cascade, as well as proteins $\mathrm{C}$ and $\mathrm{S}$ (not depicted), formed the basis of the model by Burghaus et al. (2011). its rationale in the two steps inhibited by warfarin in the hydroquinone/epoxide cycle of the vitamin $\mathrm{K}$ process (Wittkowsky, 2003). S-warfarin and R-warfarin decay with half-lives of 29 and $45 \mathrm{~h}$, respectively (Wittkowsky, 2003). Discontinuation of warfarin is modeled by simulating the decay of R-warfarin and $\mathrm{S}$-warfarin, resulting in an increase in vitamin $\mathrm{K}$-dependent factor production rates and thus factor concentrations. Figure 2 shows the obtained warfarin decay and factor increase plots over a 10 -day period $(240 \mathrm{~h})$.

The parameters R-warfarin and S-warfarin of the warfarin decay model (Table 1), which are considered to be expressed in arbitrary units of unknown proportionality to in vivo concentrations, were adjusted to reach the INR (corresponding to steady-state warfarin treatment) that was chosen as a starting point for the respective warfarin discontinuation simulation. For example, the warfarin parameter resulting in an INR of 2.5 and relative factor concentrations of approximately 30\% was found to be 0.4 (Figure 2), in line with actual clinical observations (Ferreira et al., 2002).

The warfarin action and decay model was programmed in $\mathrm{MoBi}^{\circledR} 2.3$ (Bayer Technology Services, Leverkusen, Germany) (Bayer Technology Services, 2010), as were the already published model components (see listing of model parameters in Table 1).

\section{SIMULATIONS}

Simulation of coagulation status during switching

In general, to simulate the coagulation status during a switch from warfarin to rivaroxaban, we focused on drug-drug combination effects as characterized by the PT test and trigger scenarios considered relevant for bleeding risks as well as antithrombotic efficacy (Table 2). The latter scenarios have been established and tested previously (Burghaus et al., 2011). In clinical practice PT values are commonly reported as INR values to indicate the anticoagulant effect of VKAs. Therefore, for comparison with clinical data, simulated PT test results were translated into INR values assuming an ISI (International Sensitivity Index; a characteristic of in vitro PT reagents used for calibration of experimental data) value for our in silico PT assay of 1.0.

Starting conditions for simulations of switching were set by adjusting the effective concentration of R- and S-warfarin (and the resulting steady-state concentrations of vitamin $\mathrm{K}$-dependent factors) to represent steady-state warfarin therapies at a chosen INR value, including INRs below (1.5), within (2.5), and above (3.5) the therapeutic window. For a given time point after discontinuation of warfarin therapy, the effective concentrations of Rand S-warfarin were calculated using the decay model.

To evaluate the model, we first simulated the decay of warfarin without addition of rivaroxaban and compared it with data 
Table 1 | The warfarin decay model: (A) reaction; (B) species; (C) species half-lives.

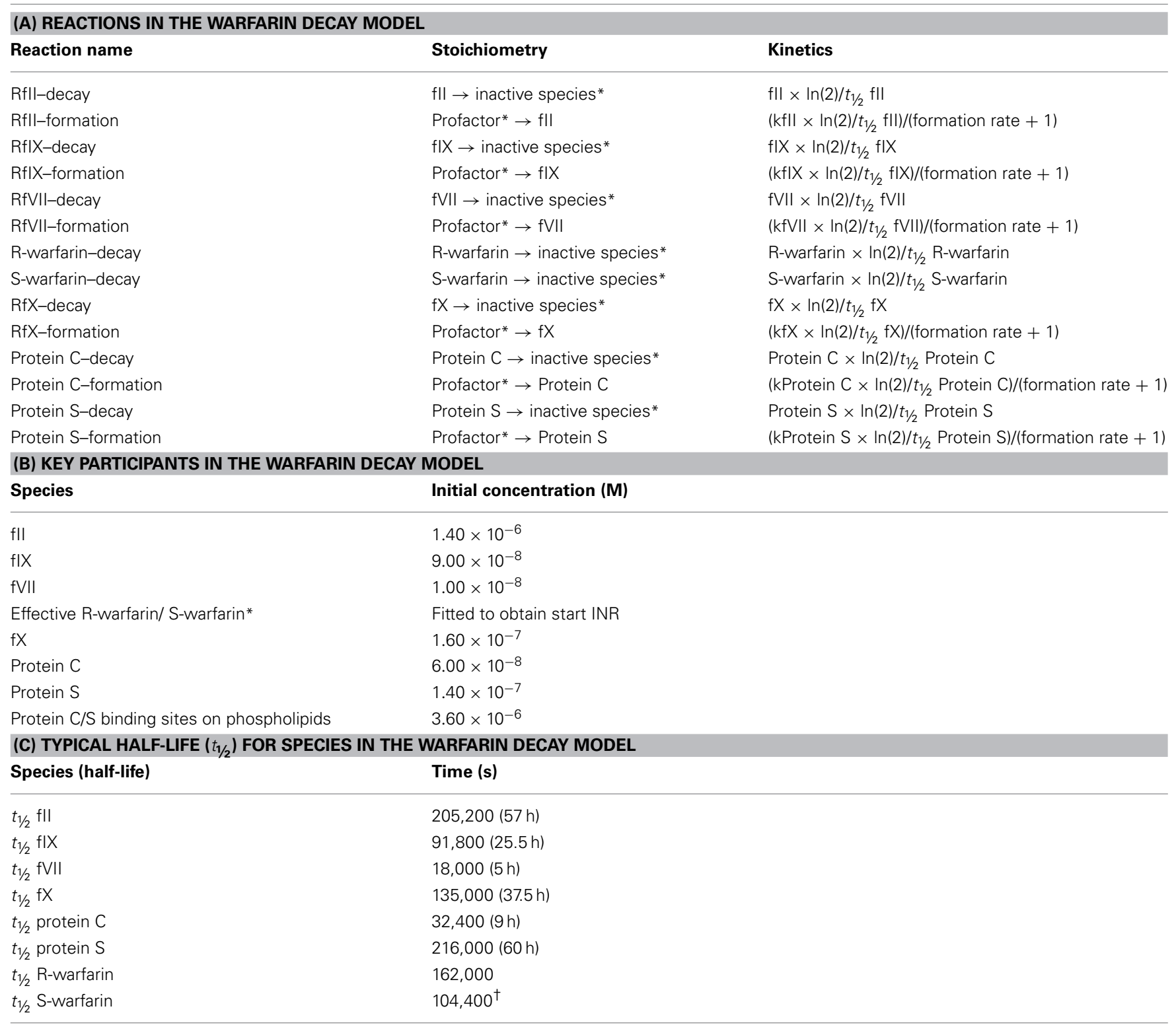

Note for Table A: For the calculation of the formation rate, see section on Turnover model for vitamin K-dependent coagulation factors in the Materials and Methods.

*Indicates a 'boundary species' that has its value kept constant during the simulation. $f$, Factor; $k$, kinetic rate constant; $R$, reaction; $t_{1 / 2}$, half-life.

Note for Table B: ${ }^{*}$ Effective $R$-warfarin and S-warfarin concentrations are in arbitrary units reflecting the fraction of remaining warfarin.

Factor concentrations (II, VII, IX, X) are average plasma concentrations taken from Hockin et al. (2002). Protein C and S concentrations were taken from Bungay et al. (2003); protein $S$ was corrected to reflect the free plasma concentration (40\% of total) reported in the protein C monograph available online at www.chromogenix. com. f, Factor; INR, international normalized ratio.

Note for Table C: ${ }^{\dagger} 74,897$ for Japanese patients. f, Factor.

from a clinical study of warfarin discontinuation. We then investigated the interaction with rivaroxaban as characterized by a PT test (and the resulting INR values) by re-running the warfarin decay simulations with added rivaroxaban, testing a broad range of rivaroxaban concentrations.

\section{Simulations of the therapeutic corridor in Caucasian patients}

To evaluate the safety and efficacy of rivaroxaban combined with residual warfarin action in virtual Caucasian patients, we used simulation to identify threshold rivaroxaban concentrations leading to the same clotting times in previously established trigger scenarios (Burghaus et al., 2011), as obtained for warfarin monotherapy at INR values of 1.5, 2.0, 4.0, and 5.0. These INR values are within the INR range considered to be of clinical relevance (Ageno et al., 2012) for the following reasons: the target therapeutic range of INRs for anticoagulation is generally between 2.0 and 3.0; special patient populations may need to be adjusted to lower INR ranges; and an INR higher than 3.0 is not uncommon in patients receiving VKA therapy. 

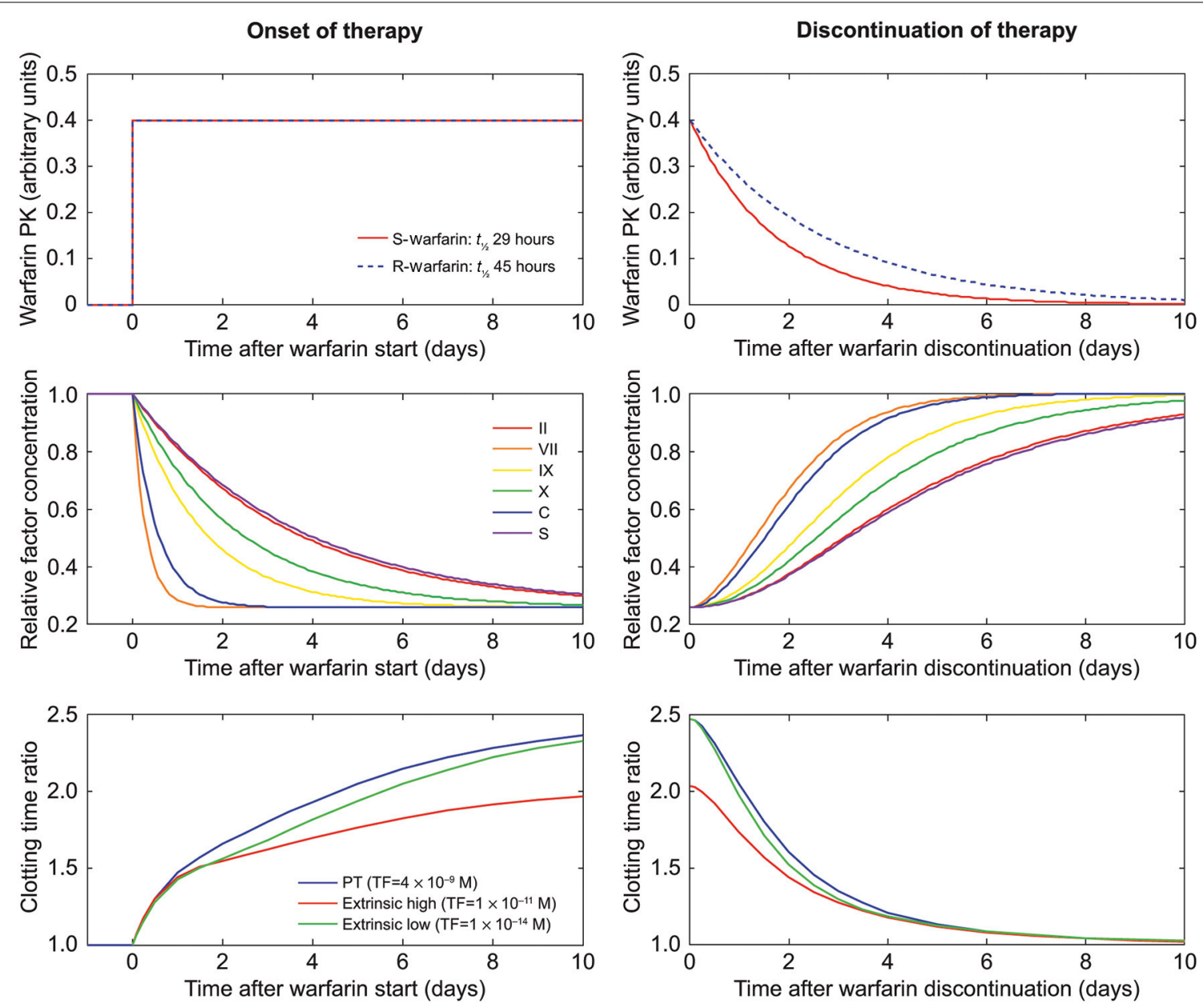

FIGURE 2 | Dynamic behavior of the warfarin decay model. Graphs on the left side show the sudden onset of warfarin therapy simulated for Caucasians. Graphs on the right side show warfarin discontinuation, eventually leading to normal values for the coagulation system. Upper graphs show the values of R-warfarin and S-warfarin (arbitrary units); middle graphs

Table 2 | Coagulation scenarios used for simulations.

\section{Scenario}

Tissue factor concentration (M)

Prothrombin time test (extrinsic)

Extrinsic strong

$$
\begin{gathered}
4.0 \times 10^{-9} \\
10^{-11} \\
10^{-14}
\end{gathered}
$$

The strong extrinsic trigger $\left(\mathrm{TF}=10^{-11} \mathrm{M}\right)$ was assumed to be the relevant safety scenario. The weak extrinsic trigger (TF $=10^{-14} \mathrm{M}$ ) was considered to be the relevant efficacy scenario. A safe rivaroxaban exposure was assumed to provide faster clotting after the strong trigger than warfarin therapies with INR values of 4.0 and 5.0 for patients with a high and a normal risk of bleeding, respectively. An efficacious rivaroxaban scenario was assumed to extend clotting times after the weak trigger beyond those times obtained for warfarin therapies at INR values of 1.5 and 2.0 for patients with normal and high thrombotic risk, respectively. show the corresponding evolution of factor concentrations; and lower graphs show coagulation time (relative to an untreated scenario) for different triggers (Table 2) in the coagulation model, depending on the factor concentrations from the middle graphs. PK, pharmacokinetics; PT, prothrombin time; TF, tissue factor; $t_{1 / 2}$, half-life.
These safety and efficacy thresholds were calculated for each time point during the first 7 days after warfarin discontinuation. The impact of INR at the time of discontinuation was evaluated by simulating initial INR values of $1.5,2.5$, and 3.5. The impact of warfarin PK variability was studied by simulating different halflives. We used typical slow, median, and fast half-lives for S- and R-warfarin $(52,29$, and $18 \mathrm{~h}$ for S-warfarin, and 70,45 , and $20 \mathrm{~h}$ for R-warfarin, respectively).

\section{Simulations of the therapeutic corridor in Japanese patients}

We also performed simulations in virtual Japanese patients. In the Japanese population, the variability of clearance via cytochrome P450 2C9 is lower than in most other populations (Takahashi et al., 2006). When simulating warfarin discontinuation for the Japanese population, we adjusted the half-life of S-warfarin by multiplying the typical values used for Caucasians with a relative clearance (469.4/654.3) for Japanese versus Caucasian patients as determined previously (Takahashi et al., 2006). The only well-documented difference in the coagulation cascade in the 
Japanese population is a reduced level of Factor VII pre-activation of $0.5 \%$ as compared with $1.0 \%$ in Caucasians (Kario et al., 1994); this difference was accounted for in the Japanese model. In the simulations of Japanese patients, R-warfarin and coagulation factor half-lives, as well as their variability ranges, were the same as for the Caucasian simulations.

\section{Impact of differences in coagulation factor expression status}

To assess the robustness of our study approach for differences in coagulation factor expression status (e.g., as a consequence of genetic predisposition), we repeated the above simulation program for a broad panel of in silico patients with different factor abnormalities (over- or under-expression in Factors I, V, VIII, and XI; Table 3 and Supplementary Material 2). These factors were chosen because they are independent of warfarin. Consequently, their levels do not vary depending on the dose of warfarin and they can be used to evaluate the sensitivity of the model.

\section{RESULTS}

To obtain initial conditions for the simulation of the discontinuation of warfarin therapy the effect of adding warfarin and its effect on factor synthesis was simulated until steady-state conditions were reached. Figure 2 illustrates the effect of setting the effective

Table 3 | Simulation program for patients with factor abnormalities.

\begin{tabular}{|c|c|c|c|c|}
\hline & Factor I & Factor V & Factor VIII & Factor XI \\
\hline Normal patient & 1 & 1 & 1 & 1 \\
\hline \multirow[t]{5}{*}{ Factor I deficient } & 0.15 & 1 & 1 & 1 \\
\hline & 0.5 & 1 & 1 & 1 \\
\hline & 0.75 & 1 & 1 & 1 \\
\hline & 2 & 1 & 1 & 1 \\
\hline & 5 & 1 & 1 & 1 \\
\hline \multirow[t]{6}{*}{ Factor V deficient } & 1 & 0.01 & 1 & 1 \\
\hline & 1 & 0.1 & 1 & 1 \\
\hline & 1 & 0.5 & 1 & 1 \\
\hline & 1 & 0.75 & 1 & 1 \\
\hline & 1 & 2 & 1 & 1 \\
\hline & 1 & 5 & 1 & 1 \\
\hline \multirow[t]{6}{*}{ Factor VIII deficient } & 1 & 1 & 0.01 & 1 \\
\hline & 1 & 1 & 0.1 & 1 \\
\hline & 1 & 1 & 0.5 & 1 \\
\hline & 1 & 1 & 0.75 & 1 \\
\hline & 1 & 1 & 2 & 1 \\
\hline & 1 & 1 & 5 & 1 \\
\hline \multirow[t]{6}{*}{ Factor XI deficient } & 1 & 1 & 1 & 0.01 \\
\hline & 1 & 1 & 1 & 0.1 \\
\hline & 1 & 1 & 1 & 0.5 \\
\hline & 1 & 1 & 1 & 0.75 \\
\hline & 1 & 1 & 1 & 2 \\
\hline & 1 & 1 & 1 & 5 \\
\hline
\end{tabular}

The values presented are ratios of normal values. warfarin concentration to 0.4 (upper left panel) on the temporal change of relative factor concentrations (middle left panel; for all three trigger scenarios) and the resulting increase in clotting times (lower left panel) over 10 days. The simulation of the discontinuation of warfarin treatment (Figure 2) showed a slow exponential decay of the effective S- and R-warfarin concentrations (Figure 2, upper right panel). Concentrations of vitamin K-dependent factors returned slowly to baseline values, defined as values in the absence of warfarin treatment (1.0; Figure 2, middle right panel), although some factors took more than 10 days to reach $100 \%$ of baseline values. Similar timescales were obtained for the decay of clotting time prolongation (Figure 2, lower right panel). Simulation data of coagulation factors were a good fit to clinical data from a warfarin discontinuation study (White et al., 1995), as shown in Figure 3.

Next, we investigated the impact of different warfarin halflives on the decay of INR (Figure 4). We simulated typical slow, median, and fast half-lives for S- and R warfarin $(52,29$, and $18 \mathrm{~h}$ for S-warfarin, and 70, 45, and $20 \mathrm{~h}$ for R-warfarin, respectively) (see Methods section) and plotted time-dependent changes in INR over 7 days (Figure 4; black lines and gray shaded area). We simulated the effect of rivaroxaban on PT and INR by adding constant concentrations of rivaroxaban to the simulated decay of warfarin action. At the time of discontinuation, a concentration of $200 \mu \mathrm{g} / \mathrm{L}$ rivaroxaban increased PT values from approximately $30 \mathrm{~s}$ owing to the warfarin effect from an INR of 2.5 to more than $50 \mathrm{~s}$ (Figure 4; red lines and shaded area), indicating a synergistic effect. Rivaroxaban alone at a concentration of $200 \mu \mathrm{g} / \mathrm{L}$ without any warfarin effect prolonged PT by $3 \mathrm{~s}$ from $12 \mathrm{~s}$ to approximately $15 \mathrm{~s}$ (Figure 4; light blue horizontal lines). A putative, only additive interaction between warfarin and rivaroxaban

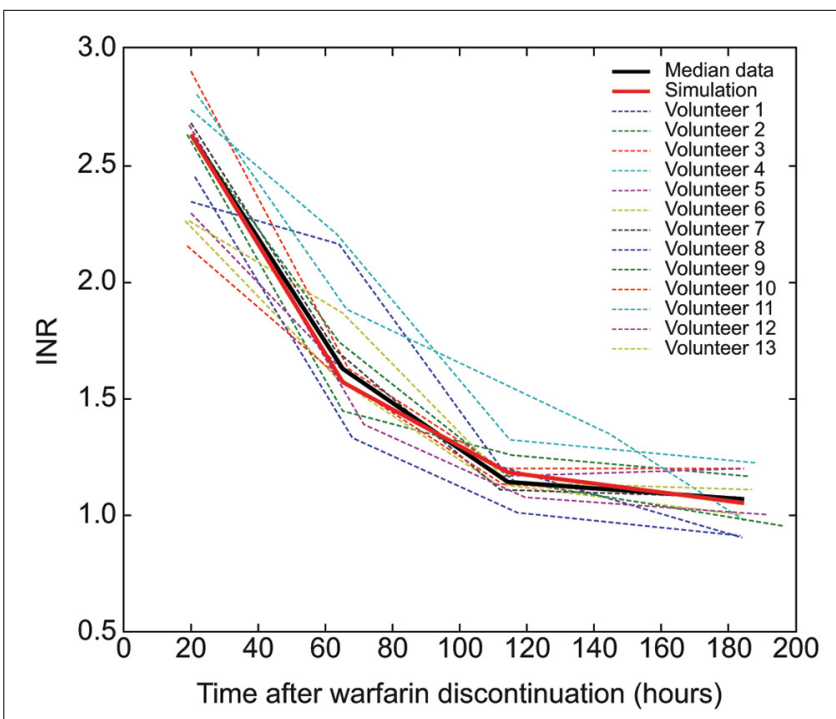

FIGURE 3 | Validation of the warfarin decay model. Thin colored lines represent the international normalized ratio (INR) data of 13 individual patients after discontinuation of warfarin therapy (White et al., 1995) (individual data courtesy of R.H. White, UC Davis, California, USA); the thick black line provides the associated median at every time point; the thick red line describes the simulated INR during warfarin decay. 


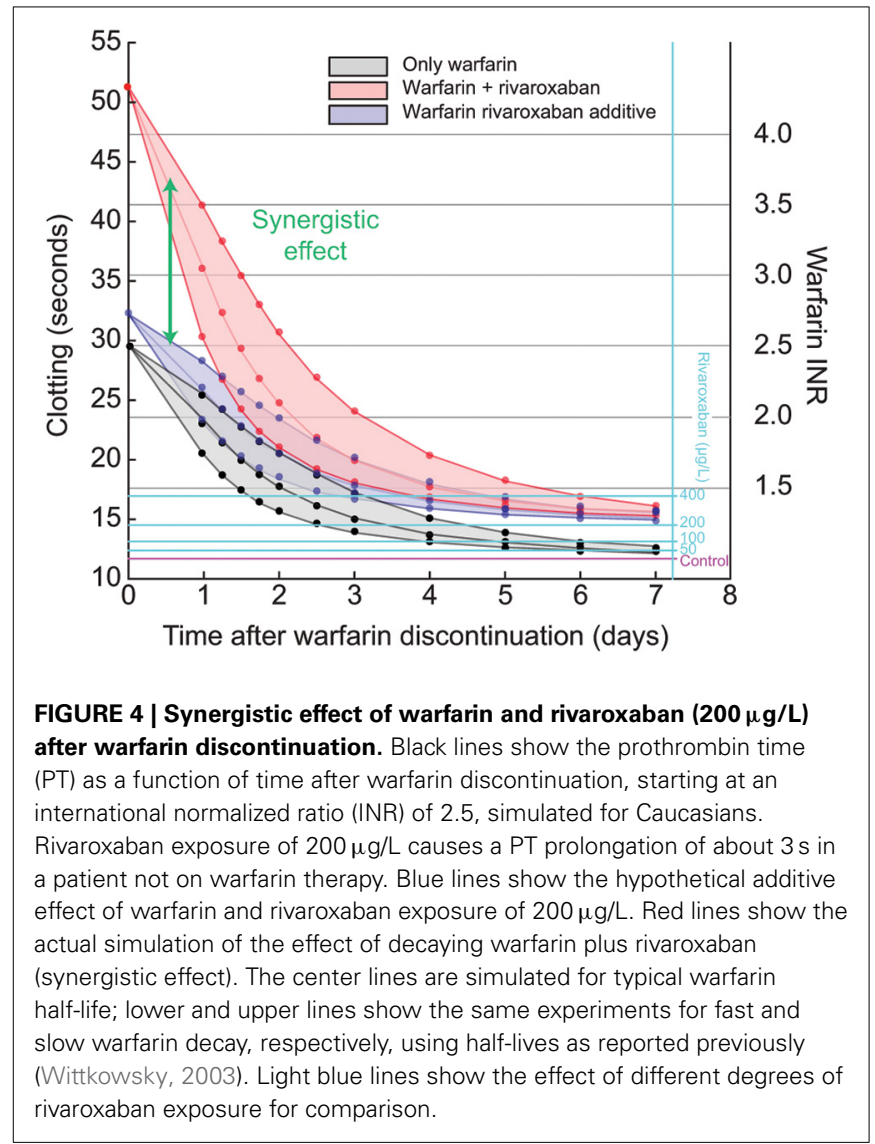

would, therefore, only explain an increase of PT from 30 to $33 \mathrm{~s}$ (Figure 4; blue lines and shaded area).

The slope of the PT versus rivaroxaban concentration relationship is a measure of rivaroxaban potency. A systematic analysis of the time dependency of this slope after warfarin discontinuation showed that the strongest synergism occurred directly after discontinuation (Figure 5A). For up to $72 \mathrm{~h}$ after discontinuation, the slope was clearly steeper than for rivaroxaban alone $(t=$ inf; Figure 5A). A similar pattern was demonstrated for simulations with Japanese in silico patients (see Materials and Methods; Figure 5B), which also showed the faster warfarin decay in these patients as a consequence of the shorter S-warfarin half-life compared with Caucasian patients (see Materials and Methods). Differences between the Japanese and Caucasian populations were observed both at the level of the relative concentrations of individual coagulation factors (Figure 5C) as well as in the resulting relative PT values (Figure 5D). These analyses showed the biggest difference between Caucasian and Japanese patients at around 2 days after warfarin discontinuation.

Next, we simulated a typical warfarin decay scenario over a 7 -day period $(168 \mathrm{~h})$ for an initial INR of 2.5 at discontinuation for Caucasian patients and calculated efficacy and safety corridors for rivaroxaban exposure. These corridors were defined by clotting time thresholds for extrinsic triggers (Table 2) as obtained for treatment with warfarin only corresponding to INR values of $1.5,2.0,4.0$, and 5.0. The results were translated into a nomogram (Figure 6) depicting the rivaroxaban concentration

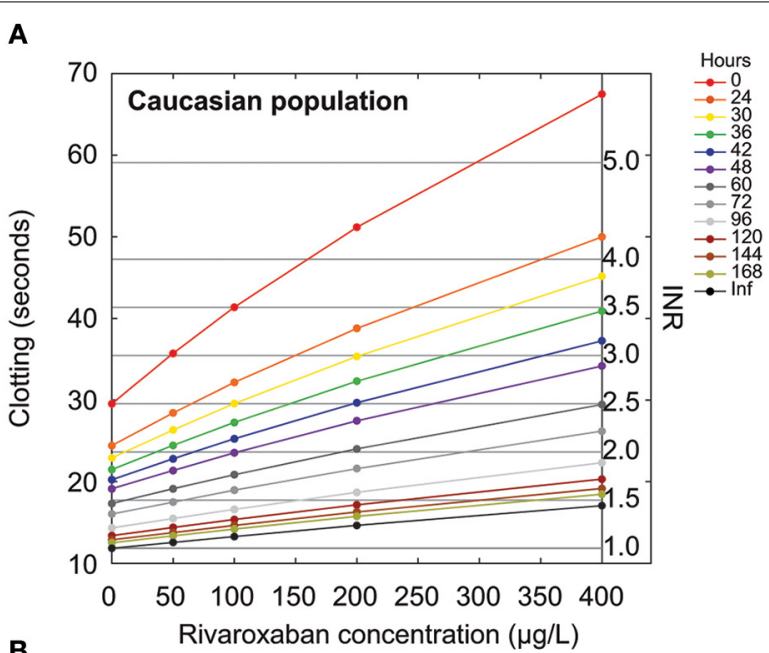

B
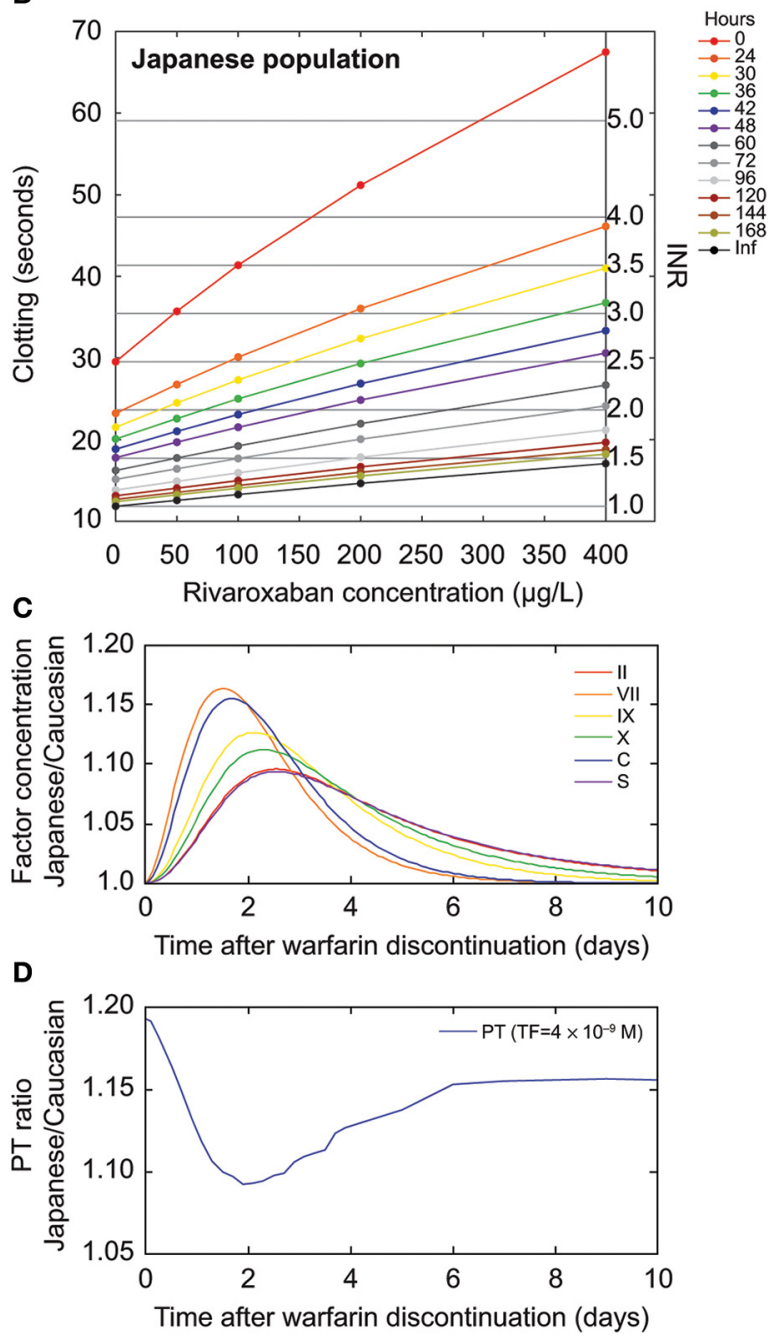

FIGURE 5 | Prothrombin time (PT) characteristics for rivaroxaban after warfarin discontinuation. (A,B) Solid black line represents the effect of rivaroxaban without warfarin. The impact of rivaroxaban is stronger when rivaroxaban therapy is started early after warfarin discontinuation. (A) Modeled for the Caucasian population. (B) Modeled for the Japanese population. (C,D) Time course of (C) vitamin K-dependent factor

(Continued) 


\section{FIGURE 5 | Continued}

concentration ratios and (D) PT ratio of simulated Japanese versus Caucasian patients. Vitamin K-dependent factors start from $40 \%$ of the normal for both Japanese and Caucasians. (D) The tissue factor (TF) value used to simulate PT was set at $4 \times 10^{-9} \mathrm{M}$. The simulated Japanese normal PT is slightly higher than the simulated Caucasian normal PT because of reduced Factor VII preactivation, resulting in a ratio $>1$ after the warfarin effect has subsided (8-10 days). Inf, infinite time (after warfarin discontinuation); INR, international normalized ratio.

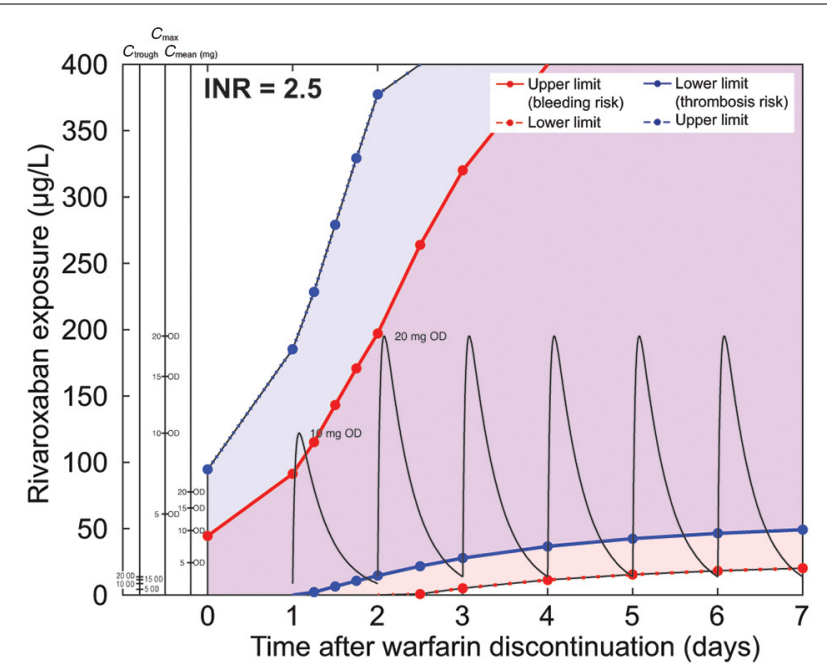

FIGURE 6 | Nomogram for a typical warfarin decay scenario starting at an international normalized ratio (INR) of $\mathbf{2 . 5}$ for the Caucasian in silico patient. The $x$-axis describes the time after discontinuation of warfarin and the $y$-axis corresponds to rivaroxaban exposure. Blue and red lines correspond to the edges of the safety and efficacy regions. The strong extrinsic trigger (TF $=10^{-11} \mathrm{M}$ ) was assumed to be the relevant safety scenario. The weak extrinsic trigger (TF $=10^{-14} \mathrm{M}$ ) was considered to be the relevant efficacy scenario. Rivaroxaban exposure above the dashed red line is considered to be safe and exposure below the dashed blue line is considered to be efficacious for most patients. The region between the solid red and solid blue lines describes the exposure range to which patients with a high risk of bleeding or thrombosis should be dosed. Patients with a high risk of bleeding should stay below the exposure limit represented by the solid red line. Patients with a high risk of thrombosis should stay above the exposure limit represented by the solid blue line. Alternative $y$-axes convert rivaroxaban exposure into mean plasma concentration $\left(C_{\text {mean }}\right)$, maximum plasma concentration $\left(C_{\max }\right)$, and minimum plasma concentration $\left(C_{\text {trough }}\right)$ values for the different rivaroxaban dosing regimens studied $(5,10,15$, and $20 \mathrm{mg}$ once daily [OD]).

Pharmacokinetic curves for rivaroxaban 10 and $20 \mathrm{mg}$ OD are drawn in black. These nomograms do not account for pharmacokinetic variability or uncertainty in parameters. However, the potential impact of pharmacokinetic variability of rivaroxaban can also be evaluated from this graph although this is not depicted explicitly.

range compliant with the calculated thresholds. The nomogram illustrates that the therapeutic window for rivaroxaban is narrower at the time of discontinuation of warfarin and widens in the subsequent days before it reaches the full width as given for monotherapy without warfarin co-medication. To relate the exposure window to rivaroxaban $\mathrm{PK}$, the $\mathrm{y}$-axis was enriched with information about trough, mean, and peak concentrations for 5 ,

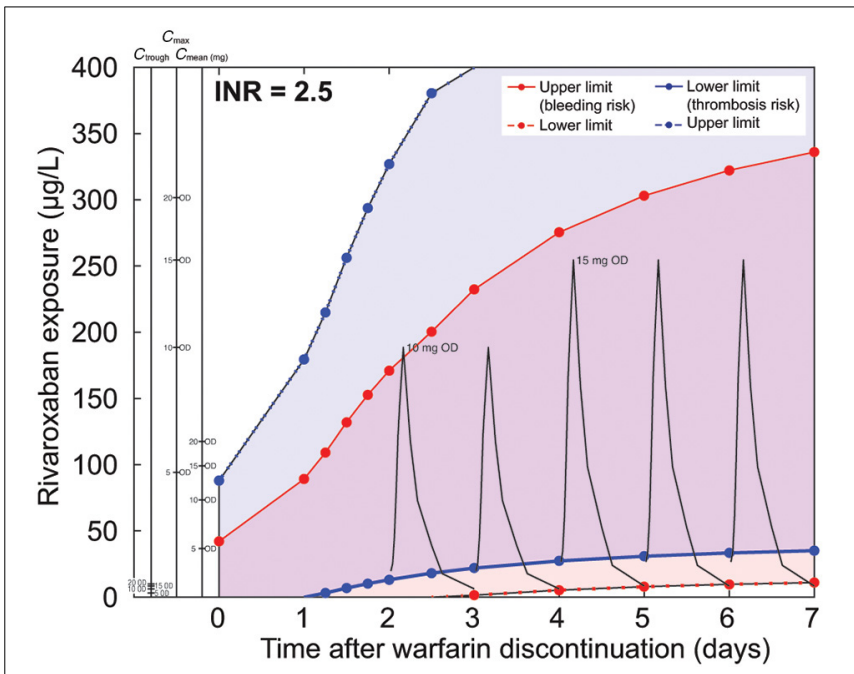

FIGURE 7 | Nomogram for a typical warfarin decay scenario starting at an international normalized ratio (INR) of $\mathbf{2 . 5}$ for the Japanese in silico patient. Pharmacokinetic curves for rivaroxaban 10 and $15 \mathrm{mg}$ OD are drawn in black. (Note: please refer to legend of Figure $\mathbf{6}$ for further details.)

10,15 , and $20 \mathrm{mg}$ once-daily doses of rivaroxaban. We plotted the mean PK profile resulting from a rivaroxaban dosing schedule starting with $10 \mathrm{mg}$ once daily on day 1 after discontinuation, and continuing with rivaroxaban $20 \mathrm{mg}$ once daily (the dose approved in Europe and the United States for the prevention of stroke and systemic embolism in patients with atrial fibrillation) (Figure 6). The superimposed PK profile shows that day-end levels were within the safety limit. Therefore, the graph demonstrates that this schedule is compliant with the in silico safety and efficacy criteria (red and blue lines, respectively).

The same type of simulation was performed for the safety and efficacy corridor for in silico Japanese patients starting at an INR of 2.5 (Figure 7). The mean rivaroxaban concentrations for two consecutive days with $10 \mathrm{mg}$ once daily starting on day 2 after warfarin discontinuation followed by rivaroxaban $15 \mathrm{mg}$ once daily doses comply with the calculated corridor. The information about mean, peak, and trough concentrations of rivaroxaban are also provided in the nomogram allowing assessment of safety and efficacy of other dosing schedules. For instance, a schedule starting on day 1 with $5 \mathrm{mg}$ once daily, continuing with a daily increase to $10 \mathrm{mg}$ once daily and finally $15 \mathrm{mg}$ once daily on day 3 , would also match the corridor.

We also investigated the effect of different baseline INR levels at discontinuation. With an INR of 1.5 at discontinuation, we found that a flat rivaroxaban schedule of $20 \mathrm{mg}$ once daily started already on day 0 , when the next warfarin dose would be due, matched the in silico criteria (Figure 8A). For comparison we have plotted the same dosing schedule as for the simulation starting at an INR of 2.5. By design, the lower efficacy threshold was above 0 immediately after discontinuation leading to the need to dose rivaroxaban in order to stay within the corridor. With an INR of 3.5 at discontinuation, the safety and efficacy corridor was very narrow just after discontinuation (Figure 8B). As for simulations with an initial INR of 2.5, the superimposed PK profile 

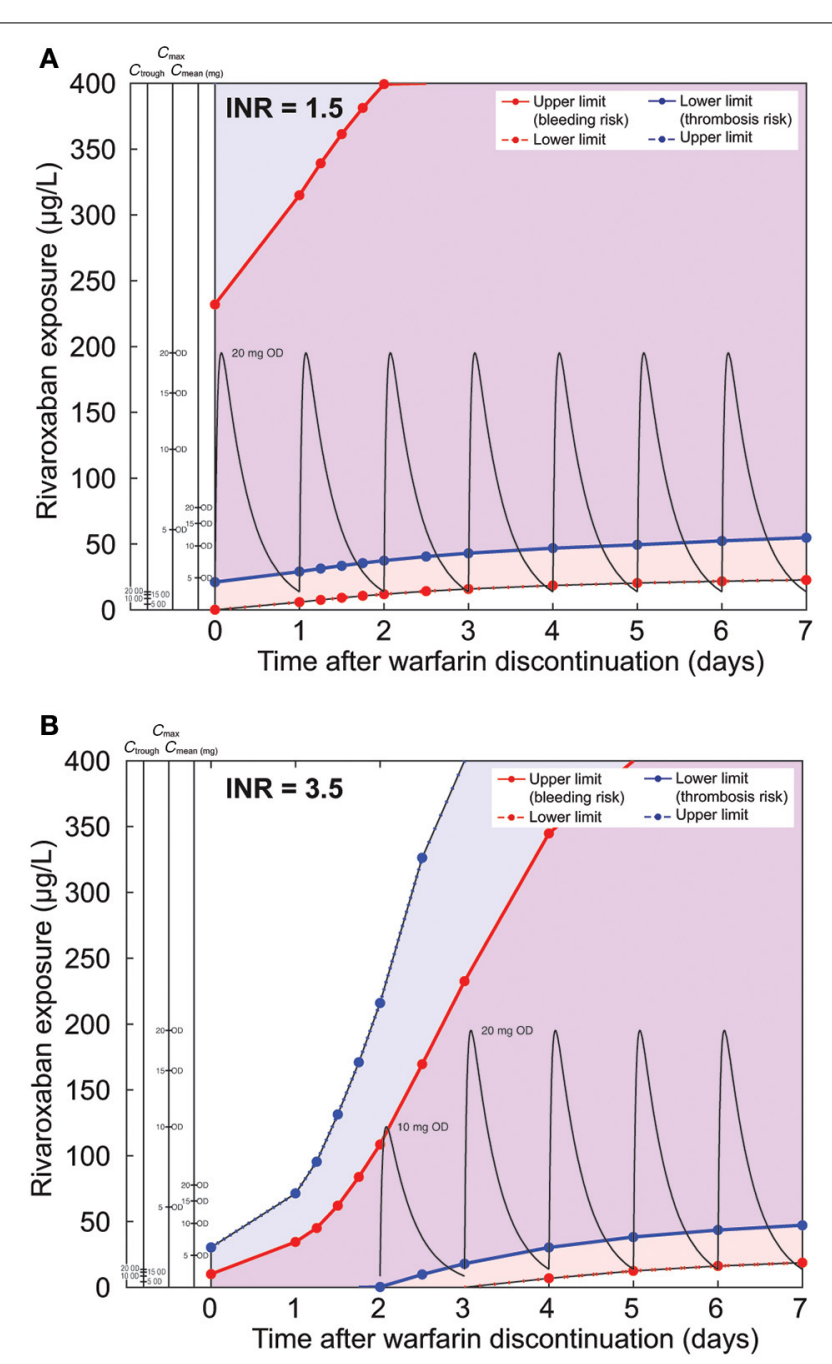

FIGURE 8 | Comparison of nomograms for patients with INR of (A) 1.5 and (B) $\mathbf{3 . 5}$ (typical warfarin decay is assumed). Pharmacokinetic curves for rivaroxaban 10 and $20 \mathrm{mg}$ OD are drawn in black. For panel (A), the dashed blue line representing the upper exposure limit for normal patients extends past the axis limits of the figure and is, therefore, not presented on the graph. (Note: please refer to legend of Figure $\mathbf{6}$ for further details.)

shows that day-end levels were within the safety limit. The nomogram suggests starting with rivaroxaban $10 \mathrm{mg}$ once daily only on day 2 after warfarin discontinuation when the therapeutic window has already broadened, followed by rivaroxaban $20 \mathrm{mg}$ once daily from day 3 onwards.

We then investigated the impact of the PK half-life of warfarin. We repeated the simulations for Caucasian patients starting at an INR of 2.5 but used half-life combinations with the slowest and fastest decay rather than the mean values used previously (Figures 6-8; mean half-lives: S-warfarin 29 h, R-warfarin 20 h). For fast decay, we used the following half-lives: S-warfarin $18 \mathrm{~h}$ and R-warfarin $45 \mathrm{~h}$ (Figure 9A). For slow decay we used halflives for S-warfarin of $52 \mathrm{~h}$ and for R-warfarin of $70 \mathrm{~h}$ (Figure 9B). As expected, a faster PK decay of warfarin broadened the safety and efficacy corridor early (Figure 9A); the dosing schedule as
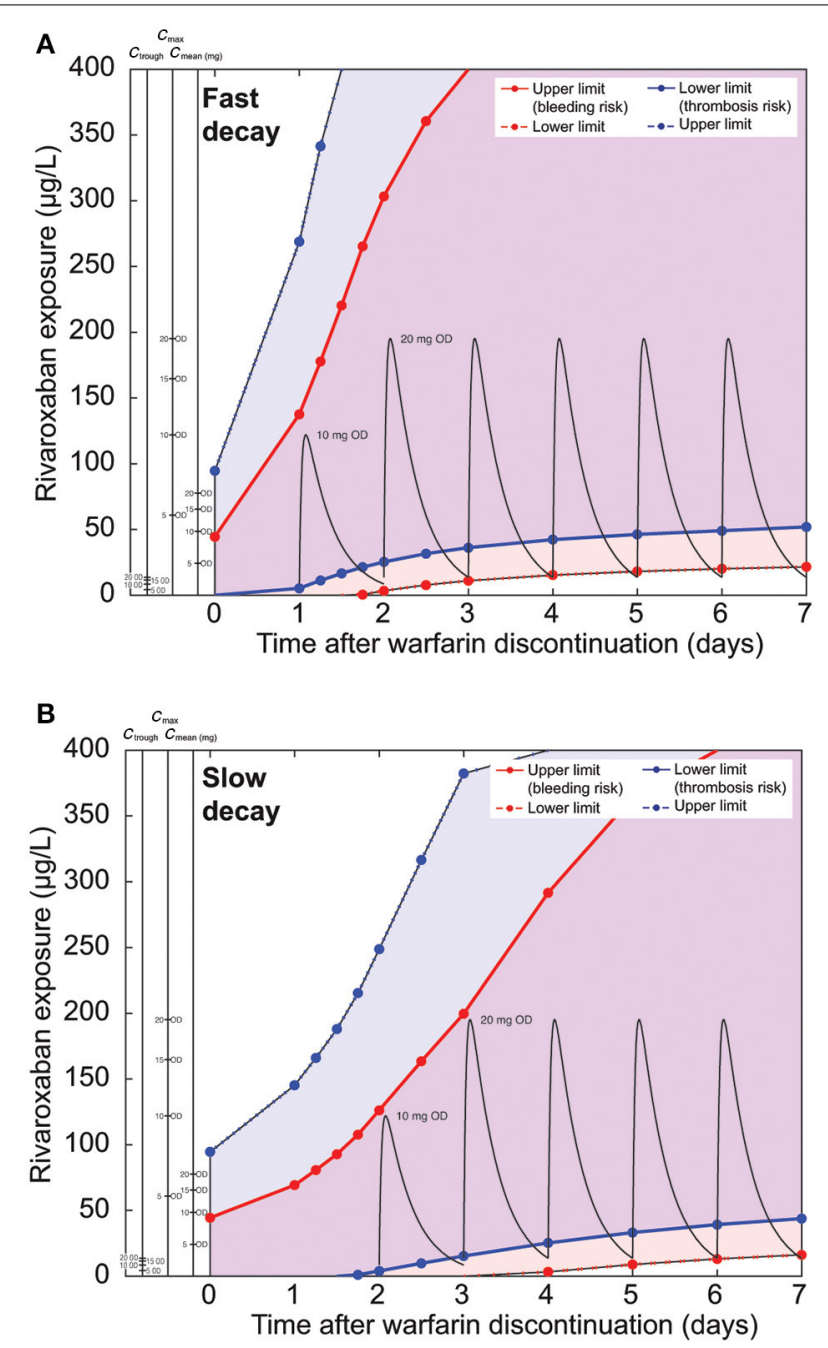

FIGURE 9 | Comparison of nomograms for patients with a starting INR of 2.5, assuming (A) fast or (B) slow warfarin decay. Pharmacokinetic curves for rivaroxaban 10 and $20 \mathrm{mg}$ OD are drawn in black. (Note: please refer to legend of Figure 6 for further details.)

suggested for normal warfarin half-lives (Figure 6) complies with the safety and efficacy corridor in this scenario. The slower decay led to a slower broadening of the corridor this time, suggesting a delayed start of rivaroxaban dosing on day 2 (Figure 9B).

Finally, we repeated the calculations for the Caucasian base case starting at an INR of 2.5 with in silico patients characterized by the over- or under-expression of various coagulation factors (Table 3; Supplementary Material 2). Only non-vitamin K-dependent factors (Factors I [fibrinogen], V, VIII, and XI) were chosen for this analysis because these can be considered to be independent of warfarin. Variations in Factors VIII or XI showed little impact on the overall appearance of the safety and efficacy corridors even at extreme over- or underexpression (Supplementary Material 2). At the lower end of the Factor I and V concentrations, the corridors were shifted toward lower levels, illustrating an overall increase in bleeding risk. At the upper extreme of Factor $\mathrm{V}$ over-expression the lower 
efficacy threshold was clearly increased. Factor I increases had little impact.

\section{DISCUSSION}

We used a computational coagulation model to investigate changes in the combined coagulation effect during the switch from warfarin to rivaroxaban in Caucasian and Japanese in silico patients. Our model was an extended version of a previously evaluated ODE-based coagulation model (Burghaus et al., 2011). We added a vitamin K-dependent factor turn-over model and an effective warfarin action and decay module. The model was structurally similar to published models by Wajima et al. (2009) and Orfeo et al. (2010, 2011). Our model also represents warfarin enantiomers and enantiomer-specific differences in warfarin clearance between Caucasian and Japanese patients (Takahashi et al., 2006). Warfarin PK is modeled via effective warfarin concentrations with respect to the resulting inhibition of the synthesis of vitamin K-dependent factors. Warfarin clearance is implemented as a decay of the inhibition of synthesis.

Our model only implicitly takes into account that the dose-response relationship of warfarin is dependent on genetic and environmental factors such as food and concomitant drugs. These factors may influence the PK (e.g., the decline of concentrations because of metabolic clearance of both S-warfarin and R-warfarin enantiomers) and the PD of warfarin (e.g., by provision of higher concentrations of vitamin $\mathrm{K}$, which counteracts VKA action; Ansell et al., 2008).

To evaluate the warfarin action and decay module, we compared the simulated decay of warfarin effect on INR with clinical data from a warfarin discontinuation study (Figure 3) (White et al., 1995) and obtained a high level of agreement. Because the model parameters were not fitted to match the clinical discontinuation data used by White et al. (1995) but taken from independent published experimental data (Wittkowsky, 2003), the excellent agreement between clinical data and the model can be considered a validation of the model extension.

When we simulated the effect of rivaroxaban exposure combined with the residual warfarin effect after discontinuation of warfarin, we demonstrated a strong synergistic effect. This finding can be explained by the fact that inhibition by warfarin leads to lower levels of Factor X, which resulted directly in lower levels of free Factor Xa and Factor Xa-Factor Va (prothrombinase complex). This inhibition is further enhanced by the fact that both its substrate thrombin and the Factor $\mathrm{X}$ activation systems (intrinsic and extrinsic tenase) are present at lower levels. Consequently, there is a higher level of inhibition when both free Factor Xa and Factor Xa-Factor Va are targeted by rivaroxaban (Perzborn et al., 2005). As expected, the synergistic effect of residual warfarin action on the rivaroxaban effect decreased over time and became negligible for a typical in silico patient after 2-3 days if the treatment with warfarin was discontinued at an INR of 2.5, in-line with guidelines for the treatment of patients with atrial fibrillation treated for the prevention of stroke (Ageno et al., 2012). For simulations with a long warfarin half-life the synergistic effect lasted longer.

To relate our simulations to clinically relevant scenarios for the switching of a patient from warfarin to rivaroxaban, we applied a set of efficacy and safety criteria. These criteria were established previously to derive the therapeutic window for rivaroxaban. The criteria were based on a comparison with warfarin effects under several simulated coagulation scenarios (Table 3 ) and allowed the identification of rivaroxaban exposure and related doses applied during the decay phase of the warfarin effect complying with the intended therapeutic effect. The outcome of the analysis was translated into nomograms for typical Caucasian and Japanese patients (Figures 6, 7), higher and lower initial INR starting values (Figure 8), and patients with high and low warfarin half-life (Figure 9). These scenarios were chosen to show robustness of the results within the range of warfarin-related variability and slightly exceeding its therapeutic range. Examples for rivaroxaban dosing schedules that fulfill the therapeutic criteria are indicated within the nomograms. Of note, although not depicted explicitly, these graphs also allow evaluation of the potential impact of pharmacokinetic variability of rivaroxaban. Depending on the simulated conditions, our analysis suggested different bridging schedules. Extreme situations such as starting at a sub-therapeutic INR value of 1.5 allowed a flat dosing of the target rivaroxaban dose for Caucasian patients (rivaroxaban $20 \mathrm{mg}$ once daily) immediately after discontinuation (Figure 8A). Simulations for typical Caucasian patients starting at an INR of 2.5 suggested starting rivaroxaban treatment with a bridging dose of $10 \mathrm{mg}$ on day 1 after discontinuation of warfarin followed by standard treatment with $20 \mathrm{mg}$ once daily from day 2 onwards. For patients starting at a higher INR value of 3.5 (Figure $8 B$ ) as well as patients starting at INR 2.5 but with a long warfarin half-life (Figure 9B), the suggested rivaroxaban schedule was shifted by 1 day.

Compared with Caucasian patients, Japanese patients have a higher exposure to rivaroxaban owing to their lower clearance via cytochrome P450 2C9 (Hori et al., 2012). Population differences in warfarin-relevant enzymes, such as vitamin $\mathrm{K}$ epoxide reductase complex subunit 1 (VKORC1) and gamma-glutamyl carboxylase (GGCX) (Kimura et al., 2007), were not explicitly included in the model but are implicitly represented by the adjustment of the initial steady-state condition in the Japanese version of the coagulation model. For typical Japanese patients (Figure 7), the suggested bridging dose on days 1 and 2 was rivaroxaban $10 \mathrm{mg}$ once daily followed by $15 \mathrm{mg}$ once daily from day 3 onwards. The lower final doses of rivaroxaban match the Japanese label (Bayer Yakuhin Ltd., 2012; Hori et al., 2012). The rationale to test the reduced dose of rivaroxaban specifically in Japanese patients was based on the higher exposure data in this population and the lower warfarin target INR in Japanese clinical practice (Hori et al., 2012). It should also be noted that the reduced dose of $15 \mathrm{mg}$ once-daily rivaroxaban is only approved in Japan but this dosing regimen may not be applicable in other Asian countries. All bridging scenarios described above (reduced bridging dose followed by standard treatment) comply with the stricter criteria for patients at high risk of bleeding. For all patients without a high risk of bleeding, flat dosing schedules starting with the target rivaroxaban dose instead of the reduced bridging dose comply with the safety and efficacy criteria.

We also simulated the effect of factor deficiencies in nonvitamin K-dependent factors (Factors I, V, VIII, and XI), because these would not necessarily be compensated for by titration of 
warfarin to a target INR. The predicted safety and efficacy corridors changed only for severe over- or under-expression of these factors (Supplementary Material 2). We conclude that this finding is only relevant for patients who previously showed clinical symptoms. This monovariate sensitivity analysis also indicated that our findings are robust against plausible variability of factor concentrations in non-hemophilic and non-thrombophilic patient populations.

We restricted our assessment to the typical simulation behavior of individual patients, representing the mean plasma PK after rivaroxaban dosing as well as typical short, intermediate, and long half-lives of warfarin. As a consequence, our simulations do not represent variability in a population of patients switching from warfarin to rivaroxaban. In clinical practice, clinicians will encounter higher as well as lower INR values in their patients. We complemented our analysis of typical behavior with an investigation of the potential impact of different (baseline) expression levels of coagulation factors not dependent on vitamin K. During model development, model parameters were selected such that they are aligned with respective (microscopic) parameters provided by the literature and that simulated systemic properties (e.g., coagulation times, amount of thrombin generated, response to different types of pharmacologic interventions) are aligned with these systemic properties reported in the literature.

Our model-based assessment provides mechanistic insight into the interaction of warfarin and rivaroxaban during the switching period between the two treatments. It supports product information for rivaroxaban in the United States and Europe (US Prescribing Information; European Summary of Product Characteristics for rivaroxaban) (Bayer Pharma, 2014; Janssen Pharmaceuticals Inc., 2014). When switching patients with atrial fibrillation from warfarin to rivaroxaban, the US Prescribing Information recommends to discontinue warfarin and if the INR is below 3.0 to start rivaroxaban on the following day (Janssen Pharmaceuticals Inc., 2014). The European Summary of Product Characteristics broadly concurs with this advice (the recommended INR is $\leq 3.0$ ). For patients treated for deep vein thrombosis and/or pulmonary embolism and for prevention of recurrent deep vein thrombosis and pulmonary embolism, the recommendation is to discontinue VKA therapy and initiate rivaroxaban when the INR is $\leq 2.5$ (Bayer Pharma, 2014). These INR-based switching strategies provide a clinically feasible approach to maximize safety and efficacy during the switching period by characterization of patients prior to switching by mean of the well-established INR value. Our simulations for initial INR values of 2.5 versus 3.5 illustrate the relevance of the INR threshold (Figures 6, 8B). In the latter case, simulations suggested initiation of rivaroxaban therapy later than day 1 after discontinuation, which would be the practical outcome of an INR value of 3.5 in the clinic. As discussed above, efficacious and safe therapies with different anticoagulants usually require different levels of biomarkers, or even different biomarkers to characterize the drug effect (for example, aPTT is a more relevant test than PT for direct thrombin inhibitors). INR is appropriate only for measuring the anticoagulant activity of VKAs. It is not appropriate for assessing anticoagulation with rivaroxaban (Bayer Pharma, 2014; Janssen Pharmaceuticals Inc., 2014) or coagulation status during the transition from warfarin to rivaroxaban (Kubitza et al., 2005a,b). The use of INR only prior to dosing of rivaroxaban consistently implements this fact.

\section{LEARNING POINTS}

The efficacy and safety nomograms defined by our simulations support the switching strategies provided in the Summary of Product Characteristics and Prescribing Information for rivaroxaban (Bayer Pharma, 2014; Janssen Pharmaceuticals Inc., 2014). There is no biomarker that predicts clinical consequences of the combined effect of rivaroxaban and warfarin in the transition phase. Large-scale clinical trials focusing on clinical outcomes of different bridging options at low or high INR in the therapeutic range of warfarin are not feasible. Simulations of different clinically realistic scenarios are presented and provide clinicians with a mechanistic pharmacologic rationale for these strategies and underline the relevance of the recommendations.

\section{ACKNOWLEDGMENTS}

The authors would like to acknowledge Stephen Purver and Claudia Wiedemann, who provided editorial support with funding from Bayer HealthCare Pharmaceuticals and Janssen Scientific Affairs, LLC. We would also like to thank Richard H. White, UC Davis, Sacramento, CA, USA, for providing the individual patient INR data for Figure 3.

\section{SUPPLEMENTARY MATERIAL}

The Supplementary Material for this article can be found online at: http://www.frontiersin.org/journal/10.3389/fphys. 2014.00417/abstract

Supplementary Material 1 | Information pack including specification of model parameters and codes used for the reproduction of study simulations. Simulations were generated using the following versions of the BTS software platform: $\mathrm{MoBi}^{\circledR}$ 2.3, MoBi ${ }^{\circledR}$ Toolbox for Matlab ${ }^{\circledR}$ 2.3.

This pack provides information on how to download the software, how to re-run the simulations and how to reproduce the graphical representations.

\section{Supplementary Material 2 | Nomograms for in silico patients with different factor abnormalities, including over-expression and under-expression of the non-vitamin K-dependent factors I, V, VIII, and XI.} Factor deficiencies are indicated by $\mathrm{x}$-fold change compared with normal values and range from 0.01 to 5 . The $x$-axis describes the time after discontinuation of warfarin and the $y$-axis corresponds to rivaroxaban exposure. Blue and red lines correspond to the edges of the safety and efficacy region. The strong extrinsic trigger (TF $=10^{-11} \mathrm{M}$ ) was assumed to be the relevant safety scenario. The weak extrinsic trigger $\left(T F=10^{-14} \mathrm{M}\right.$ ) was considered to be the relevant efficacy scenario. Rivaroxaban exposure above the dashed red line is considered to be safe and exposure below the dashed blue line is considered to be efficacious for most patients. The region between the solid red and solid blue lines describes the exposure range to which patients with a high risk of bleeding or thrombosis should be dosed. Patients with a high risk of bleeding should stay below the exposure limit represented by the solid red line. Patients with a high risk of thrombosis should stay above the exposure limit represented by the solid blue line. Alternative $y$-axes convert rivaroxaban exposure into mean plasma concentration $\left(C_{\text {mean }}\right)$, 
maximum plasma concentration $\left(C_{\max }\right)$, and minimum plasma concentration ( $\left.C_{\text {trough }}\right)$ values for the different dosing regimens studied $(5$, 10,15 , and $20 \mathrm{mg}$ once daily [OD]). These nomograms do not account for pharmacokinetic variability or uncertainty in parameters. However, the potential impact of pharmacokinetic variability of rivaroxaban can also be evaluated from these graphs although this is not depicted explicitly. To extract text information from the figure panels, such as labels of $x$-and $y$-axes or other parameters, please zoom into the panel of interest. INR, international normalized ratio.

\section{REFERENCES}

Ageno, W., Gallus, A. S., Wittkowsky, A., Crowther, M., Hylek, E. M., and Palareti, G. (2012). Oral anticoagulant therapy: antithrombotic therapy and prevention of thrombosis, 9th ed: American College of Chest Physicians evidence-based clinical practice guidelines. Chest 141, e44S-e88S. doi: 10.1378/chest.11-2292

Anand, M., Rajagopal, K., and Rajagopal, K. R. (2003). A model incorporating some of the mechanical and biochemical factors underlying clot formation and dissolution in flowing blood. J. Theor. Med. 5, 183-218. doi: 10.1080/10273660412331317415

Ansell, J., Hirsh, J., Hylek, E., Jacobson, A., Crowther, M., and Palareti, G. (2008). Pharmacology and management of the vitamin K antagonists: American College of Chest Physicians evidence-based clinical practice guidelines (8th Edition). Chest 133, 160S-198S. doi: 10.1378/chest.08-0670

Bayer Pharma, A. G. (2014). Xarelto ${ }^{\circledR}$ (rivaroxaban) Summary of Product Characteristics. Available online at: http://www.ema.europa.eu/ docs/en_GB/document_library/EPAR_-_Product_Information/human/000944/ WC500057108.pdf

Bayer Technology Services. (2010). MoBI Software Information. Available online at: www.systems-biology.com/mobi

Bayer Yakuhin Ltd. (2012). Xarelto (rivaroxaban) Japanese package insert. Available at: http://www.mhlw.go.jp/file/05-Shingikai-11121000-IyakushokuhinkyokuSoumuka/0000015162.pdf

Bungay, S. D., Gentry, P. A., and Gentry, R. D. (2003). A mathematical model of lipid-mediated thrombin generation. Math. Med. Biol. 20, 105-129. doi: 10.1093/imammb/20.1.105

Burghaus, R., Coboeken, K., Gaub, T., Kuepfer, L., Sensse, A., Siegmund, H. U., et al. (2011). Evaluation of the efficacy and safety of rivaroxaban using a computer model for blood coagulation. PLoS ONE 6:e17626. doi: 10.1371/journal.pone.0017626

Douketis, J. D., Berger, P. B., Dunn, A. S., Jaffer, A. K., Spyropoulos, A. C., Becker, R. C., et al. (2008). The perioperative management of antithrombotic therapy: American College of Chest Physicians evidence-based clinical practice guidelines (8th Edition). Chest 133, 299S-339S. doi: 10.1378/chest.08-0675

Faaij, R. A., Burggraaf, J., Schoemaker, R. C., Van Amsterdam, R. G., and Cohen, A. F. (2002). Absence of an interaction between the synthetic pentasaccharide fondaparinux and oral warfarin. Br. J. Clin. Pharmacol. 54, 304-308. doi: 10.1046/j.1365-2125.2002.01616.x

Faaij, R. A., van Griensven, J. M., Schoemaker, R. C., Goggin, T., Guenzi, A., Kroon, J. M., et al. (2001). The effect of warfarin on the pharmacokinetics and pharmacodynamics of napsagatran in healthy male volunteers. Eur. J. Clin. Pharmacol. 57, 25-29. doi: 10.1007/s002280100270

Ferreira, C. N., Vieira, L. M., Dusse, L. M., Reis, C. V., Amaral, C. F., Esteves, W. A., et al. (2002). Evaluation of the blood coagulation mechanism and platelet aggregation in individuals with mechanical or biological heart prostheses. Blood Coagul. Fibrinolysis 13, 129-134. doi: 10.1097/00001721-200203000-00008

Food and Drug Administration. (2011). FDA Draft Briefing Document for the Cardiovascular and Renal Drugs Advisory Committee (CRDAC). Food and Drug Administration. Available online at: http:/www.fda.gov/downloads/ AdvisoryCommittees/CommitteesMeetingMaterials/drugs/Cardiovascularand RenalDrugsAdvisoryCommittee/ucm270796.pdf

Hockin, M. F., Jones, K. C., Everse, S. J., and Mann, K. G. (2002). A model for the stoichiometric regulation of blood coagulation. J. Biol. Chem. 277, 18322-18333. doi: 10.1074/jbc.M201173200

Hori, M., Matsumoto, M., Tanahashi, N., Momomura, S., Uchiyama, S., Goto, S., et al. (2012). Rivaroxaban vs. warfarin in Japanese patients with atrial fibrillation - the J-ROCKET AF study. Circ. J. 76, 2104-2111. doi: 10.1253/circj.CJ-12-0454
Janssen Pharmaceuticals Inc. (2014). Xarelto ${ }^{\circledR} \quad$ (rivaroxaban) Prescribing Information. Available online at: http://www.xareltohcp.com/ sites/default/files/pdf/xarelto_0.pdf

Johnson and Johnson Pharmaceutical Research and Development. (2011). Advisory Committee Briefing Document: Rivaroxaban for the Prevention of Stroke and Non-Central Nervous System (CNS) Systemic Embolism in Patients with Atrial Fibrillation. Available online at: http://www.fda.gov/downloads/advisorycommittees/committeesmeetingmateri als/drugs/cardiovascularandrenaldrugsadvisorycommittee/ucm270797.pdf

Kario, K., Miyata, T., Sakata, T., Matsuo, T., and Kato, H. (1994). Fluorogenic assay of activated factor VII. Plasma factor VIIa levels in relation to arterial cardiovascular diseases in Japanese. Arterioscler. Thromb. 14, 265-274. doi: 10.1161/01.ATV.14.2.265

Kearon, C., Akl, E. A., Comerota, A. J., Prandoni, P., Bounameaux, H., Goldhaber, S. Z., et al. (2012). Antithrombotic therapy for VTE disease: antithrombotic therapy and prevention of thrombosis, 9th ed: American College of Chest Physicians evidence-based clinical practice guidelines. Chest 141, e419S-e494S. doi: 10.1378/chest.11-2301

Kimura, R., Miyashita, K., Kokubo, Y., Akaiwa, Y., Otsubo, R., Nagatsuka, K., et al. (2007). Genotypes of vitamin K epoxide reductase, gamma-glutamyl carboxylase, and cytochrome P450 2C9 as determinants of daily warfarin dose in Japanese patients. Thromb. Res. 120, 181-186. doi: 10.1016/j.thromres.2006. 09.007

Kogan, A. E., Kardakov, D. V., and Khanin, M. A. (2001). Analysis of the activated partial thromboplastin time test using mathematical modeling. Thromb. Res. 101, 299-310. doi: 10.1016/S0049-3848(00)00405-9

Kubitza, D., Becka, M., Voith, B., Zuehlsdorf, M., and Wensing, G. (2005a). Safety, pharmacodynamics, and pharmacokinetics of single doses of BAY 59-7939, an oral, direct Factor Xa inhibitor. Clin. Pharmacol. Ther. 78, 412-421. doi: 10.1016/j.clpt.2005.06.011

Kubitza, D., Becka, M., Wensing, G., Voith, B., and Zuehlsdorf, M. (2005b). Safety, pharmacodynamics, and pharmacokinetics of BAY 59-7939 - an oral, direct Factor Xa inhibitor - after multiple dosing in healthy male subjects. Eur. J. Clin. Pharmacol. 61, 873-880. doi: 10.1007/s00228-005-0043-5

Orfeo, T., Butenas, S., Brummel-Ziedins, K. E., Gissel, M., and Mann, K. G. (2010). Anticoagulation by factor Xa inhibitors. J. Thromb. Haemost. 8, 1745-1753. doi: 10.1111/j.1538-7836.2010.03917.x

Orfeo, T., Gissel, M., Butenas, S., Undas, A., Brummel-Ziedins, K. E., and Mann, K. G. (2011). Anticoagulants and the propagation phase of thrombin generation. PLoS ONE 6:e27852. doi: 10.1371/journal.pone. 0027852

Orfeo, T., and Mann, K. G. (2005). Mathematical and biological models of blood coagulation. J. Thromb. Haemost. 3, 2397-2398. doi: 10.1111/j.15387836.2005.01592.x

Patel, M. R., Mahaffey, K. W., Garg, J., Pan, G., Singer, D. E., Hacke, W., et al. (2011). Rivaroxaban versus warfarin in nonvalvular atrial fibrillation. N. Engl. J. Med. 365, 883-891. doi: 10.1056/NEJMoa1009638

Perzborn, E., Strassburger, J., Wilmen, A., Pohlmann, J., Roehrig, S., Schlemmer, K. H., et al. (2005). In vitro and in vivo studies of the novel antithrombotic agent BAY 59-7939 - an oral, direct Factor Xa inhibitor. J. Thromb. Haemost. 3, 514-521. doi: 10.1111/j.1538-7836.2005.01166.x

Reiffel, J. A. (2004). The potential for changing prescribing patterns from warfarin to oral direct thrombin inhibitors: clinical scenarios. Rev. Cardiovasc. Med. 5(Suppl. 5), S12-S21.

Roehrig, S., Straub, A., Pohlmann, J., Lampe, T., Pernerstorfer, J., Schlemmer, K. H., et al. (2005). Discovery of the novel antithrombotic agent 5-chloro-N-([(5S)-2-oxo-3-[4-(3-oxomorpholin-4-yl)phenyl]-1,3-oxazolidin5-yl]methyl)thiophene-2-carboxamide (BAY 59-7939): an oral, direct Factor Xa inhibitor. J. Med. Chem. 48, 5900-5908. doi: 10.1021/ jm050101d

Takahashi, H., Wilkinson, G. R., Nutescu, E. A., Morita, T., Ritchie, M. D., Scordo, M. G., et al. (2006). Different contributions of polymorphisms in VKORC1 and CYP2C9 to intra- and inter-population differences in maintenance dose of warfarin in Japanese, Caucasians and African-Americans. Pharmacogenet. Genomics 16, 101-110. doi: 10.1097/01.fpc.0000184955. 08453.a8

Wajima, T., Isbister, G. K., and Duffull, S. B. (2009). A comprehensive model for the humoral coagulation network in humans. Clin. Pharmacol. Ther. 86, 290-298. doi: $10.1038 / \mathrm{clpt} .2009 .87$ 
White, R. H., McKittrick, T., Hutchinson, R., and Twitchell, J. (1995). Temporary discontinuation of warfarin therapy: changes in the international normalized ratio. Ann. Intern. Med. 122, 40-42. doi: 10.7326/0003-4819-122-1-19950101000006

Wittkowsky, A. K. (2003). Warfarin and other coumarin derivatives: pharmacokinetics, pharmacodynamics, and drug interactions. Semin. Vasc. Med. 3, 221-230. doi: 10.1055/s-2003-44457

Conflict of Interest Statement: Rolf Burghaus, Anke Sensse, Wolfgang Mueck, Takahiko Tanigawa, and Jörg Lippert are employed by Bayer Pharma AG. Katrin Coboeken, Thomas Gaub, Christoph Niederalt, Hans-Ulrich Siegmund, and Wolfgang Weiss are employed by Bayer Technology Services GmbH.

Received: 14 June 2013; accepted: 09 October 2014; published online: 07 November 2014.
Citation: Burghaus R, Coboeken K, Gaub T, Niederalt C, Sensse A, Siegmund $H$-U, Weiss W, Mueck W, Tanigawa T and Lippert J (2014) Computational investigation of potential dosing schedules for a switch of medication from warfarin to rivaroxaban - an oral, direct Factor Xa inhibitor. Front. Physiol. 5:417. doi: 10.3389/ fphys.2014.00417

This article was submitted to Computational Physiology and Medicine, a section of the journal Frontiers in Physiology.

Copyright (C) 2014 Burghaus, Coboeken, Gaub, Niederalt, Sensse, Siegmund, Weiss, Mueck, Tanigawa and Lippert. This is an open-access article distributed under the terms of the Creative Commons Attribution License (CCBY). The use, distribution or reproduction in other forums is permitted, provided the original author(s) or licensor are credited and that the original publication in this journal is cited, in accordance with accepted academic practice. No use, distribution or reproduction is permitted which does not comply with these terms. 